
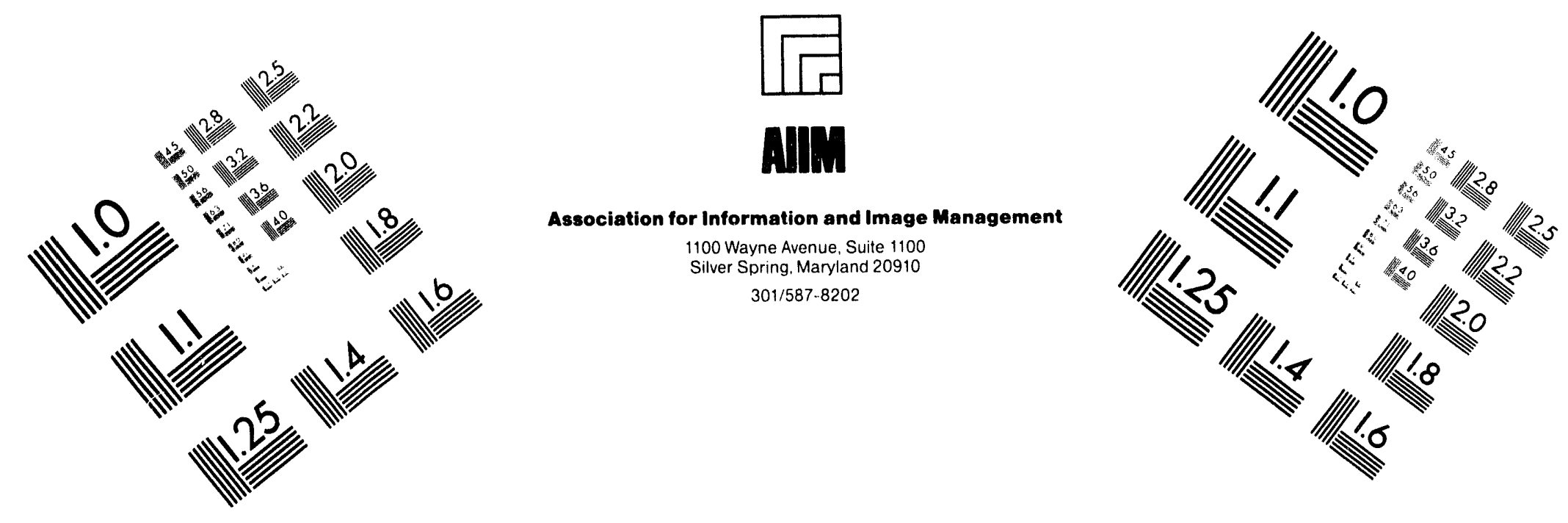

\title{
Centimeter
}

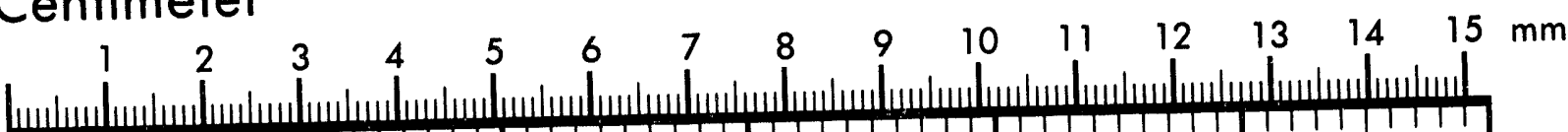

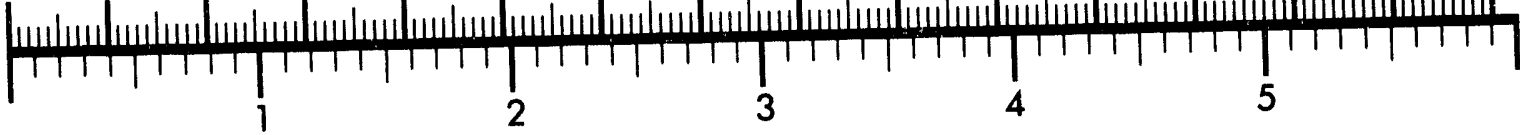
Inches
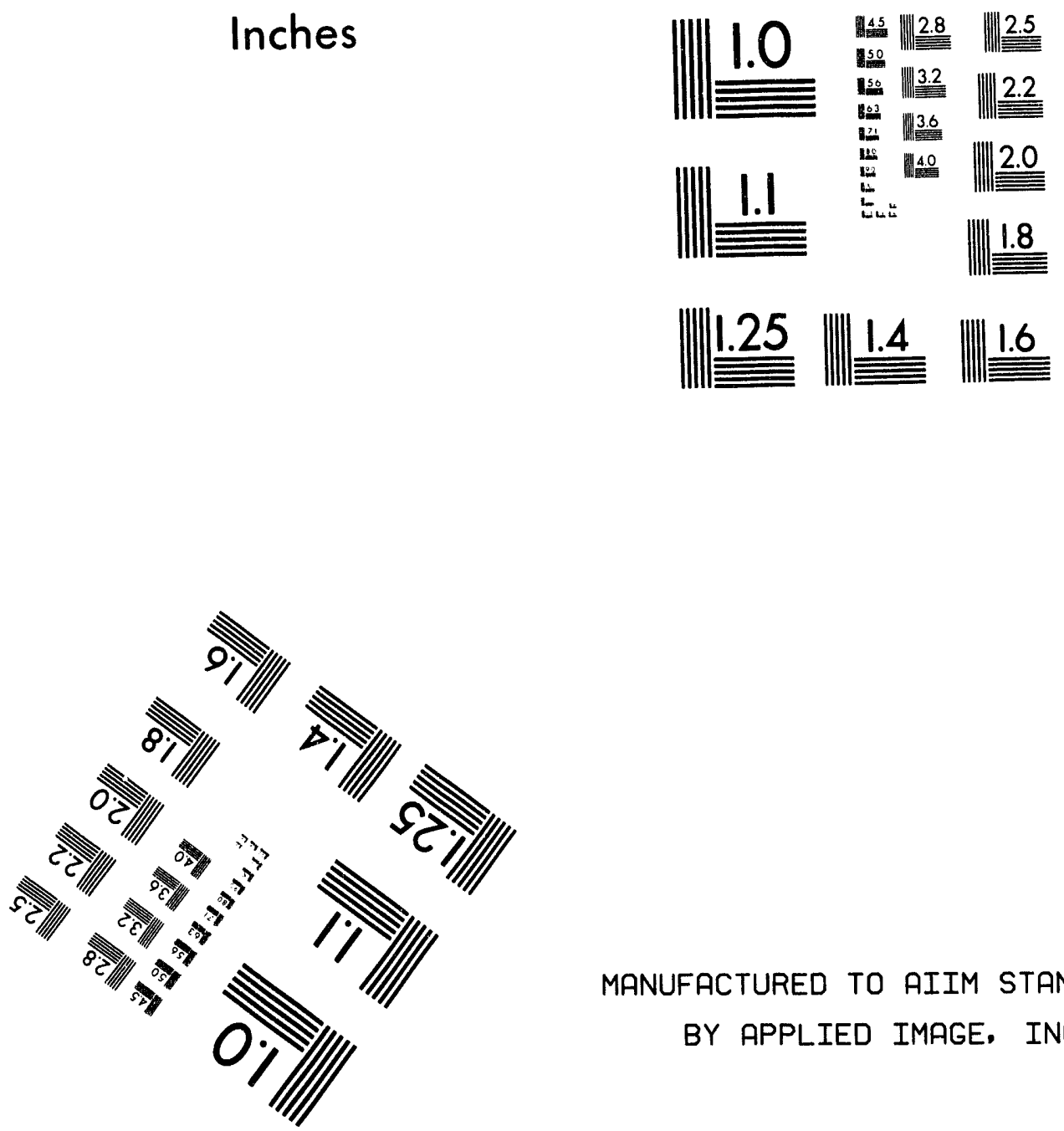

MANUFACTURED TO AIIM STANDARDS

BY APPLIED IMAGE, INC.

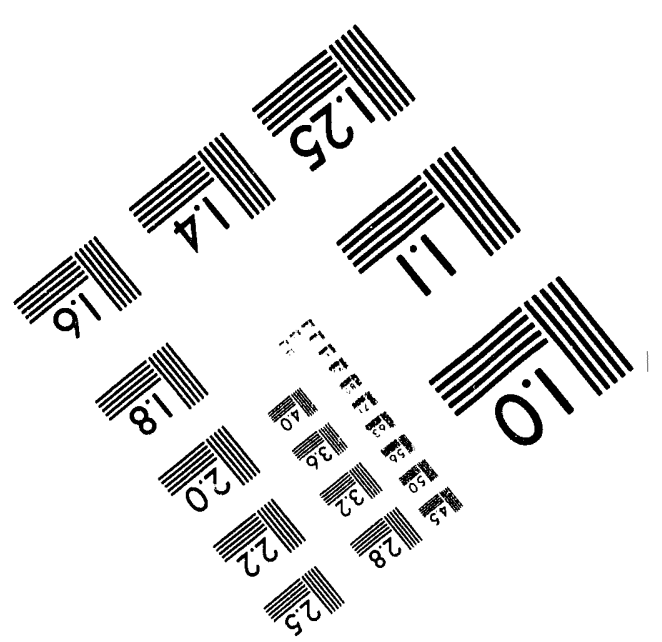



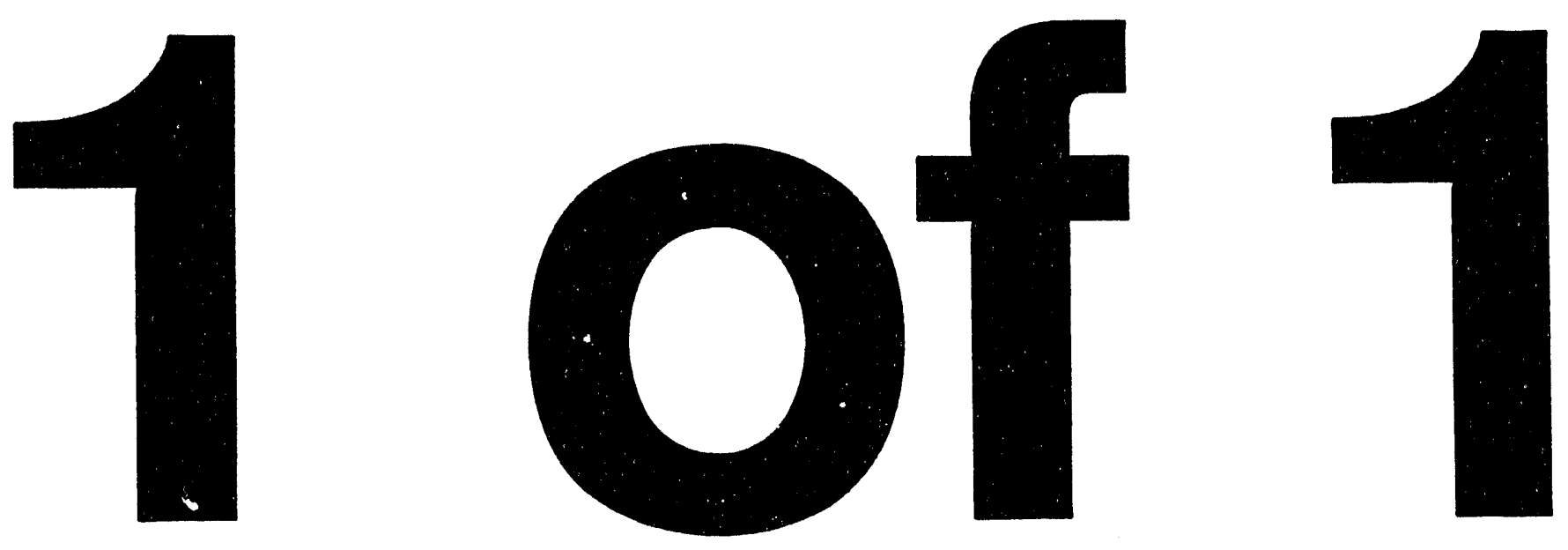


\title{
Conf. $9406201--2$
}

UCRL-JC-117539

PREPRINT

\section{A Structural Determination using Magnetic X-Ray Circular Dichroism in Spin-Polarized Photoelectron Diffraction}

\author{
G.D. Waddill \\ J.G. Tobin \\ $X$. Guo \\ S.Y. Tong
}

This paper was prepared for submittal to the journal "Vacuum" and

Conference Proceedings, 4th European Vacuum Conference and

1st Swedish Vacuum Meeting

Uppsala, Sweden

June 13-17, 1994

May 26, 1994

This is a preprint of a paper intended for publication in a joumal or proceed inga. Since changes may be made before publication, this preprint is made available with the underatanding that it will not be cited or reproduced without the permiesion of the author. 


\section{DISCLAIMER}

This document was prepared $s=n$ account of work sponsored by $m$ agency of the United States Government. Neither the United States Government nor the Uni versity of California nor any of their employees, makes any warranty, express or inplied, or assumes any legal liability or responsibility for the accuracy, completeness, or usefulness of any information, apparatus, product, or process disclosed, or represents that its use would not infringe privalely owmed rights. Reference herein to any specific commercial products, process, or service by trade name, trademark, manufacturer, or otherwise, does not necessarily constitute or imply its endorsement, recommendation, or favoring by the United States Government or the University of California. The views and opinions of authors expressed herein do not necessarily state or reflect those of the United States Government or the University of Califomia, and shall not be used for advertising or product endorsement purposes. 


\title{
A Structural Determination using Magnetic X-Ray Circular Dichroism in Spin-Polarized Photoelectron Diffraction
}

\author{
G.D. Waddill and J.G. Tobin \\ Lawrence Livermore National Laboratory \\ Chemistry and Materials Science Department \\ P.0. Box 808, L-357, Livermore, CA 94550 - USA \\ and \\ X. Guo and S.Y. Tong \\ University of Wisconsin-Milwaukee \\ Laboratory for Surface Studies and Department of Physics \\ Milwaukee, MI 53201 - USA
}

\begin{abstract}
The first structural determination with spin-polarized, energy-dependent photoelectron diffraction using circularly-polarized $x$-rays is reported for Fe films on $\mathrm{Cu}(001)$. Circularly-polarized $x$-rays produce spin-polarized photoelectrons from the Fe $2 \mathrm{p}$ doublet, and intensity asymmetries in the $2 p_{3 / 2}$ level are observed. Fully spin-specific multiple scattering calculations reproduce the experimentally-determined energy and angular dependences. A new analytical procedure which and focuses upon intensity variations due to spin-dependent diffraction is introduced.
\end{abstract}

PACS: 61.14.-x, 61.14.Dc, 75.25.+z, 75.70.Ak 
Determining the nanoscale structure-property relationships of a magnetic surface, ultrathin film or interface remains a formidable but potentially extraordinarily rewarding task. For example, as studies of giant magneto resistive ${ }^{1}$ and spin valve ${ }^{2}$ systems have progressed, it has become clear that spin-specific interfacial scattering is a critical but poorly understood event. To properly address such problems, a probe which combines elemental specificity with a sensitivity to local order, both geometric and magnetic, is essential. Spin-polarized photoelectron diffraction (SPPD) is potentially an ideal candidate for studying nanoscale magnetic systems, with the above attributes plus the promise of an ultimate extension to element-and spin-specific imaging ${ }^{3}$. However, while the potential for SPPD is very high, particularly with the advent of third generation synchrotron radiation facilities such as the Advanced Light Source, until now a definitive demonstration has been lacking. This is primarily because of the relatively inefficient nature of spin-detectors and the relatively weak nature of magnetic scattering effects. Here we present a prototype study using magnetic $x$-ray circular dichroism, to permit a direct and unambiguous control of electron polarization without the necessity of the low-efficiency spindetectors. This is coupled with a fully spin-specific, multiple scattering computational analysis. While we remained hampered by the limitations of existing sources of circularly-polarized $x$-rays and hence are constrained in terms of signal to noise, this is the first definitive demonstration of SPPD.

To provide a stringent test of SPPD, we chose to use $\mathrm{Fe} / \mathrm{Cu}(001)$. This model system exhibits the perpendicular magnetization 4 that may be used in higher density magnetic recording media. Additionally, $\mathrm{Fe} / \mathrm{Cu}(001)$ has been highly studied and the subject of considerable interest ${ }^{5-10}$ : in fact, we reproduced the forward focusing photoelectron diffraction (PD) results of Chambers, et al. 5 as an in situ cross-check of alignment. $\mathrm{Fe} / \mathrm{Cu}(001)$ at low temperatures and low coverages is an fcc structure, thus exhibiting the template effects so crucial to manipulation of ultrathin properties plus providing a classical test of the sensitivity of photoelectron diffraction to local order.

In some respects, this SPPD investigation is an independent verification and extension of the pioneering studies of G. Schütz, et al. ${ }^{11}$, who used spin-polarized EXAFS to probe bulk magnetic systems. Consistent with non-spin PD and EXAFS studies, the SPPD shows a larger effect: the SPPD oscillations are on the order of $2 \%$ while the Gd metal SPEXAFS oscillations are $\leq 1 / 3 \%$. Additionally, SPPD has the advantage of both energy and angular variations, which is essential to the extension to photoelectron diffraction imaging ${ }^{3}$. Both this work and the ground-breaking studies of Schütz, et al. ${ }^{11}$ are predicated upon control of spin-polarization of ejected electrons via excitation with circularly-polarized $x$-rays. In a simplistic picture, $2 p$ 
photoemission total cross sections from ferromagnetic materials will exhibit a polarized distribution of $62.5 \%$ (37.5\%) minority spin electrons from the $2 \mathrm{p} 3 / 2$ and $25 \%(75 \%)$ minority spin electrons from the $2 \mathrm{p}_{1 / 2}$, when excited with right (left) circularly-polarized radiation that is collinear with the magnetic axis of the sample. These adjustably spin-polarized electrons can then scatter off of nearby neighbors, producing a sensitivity to both local geometric and magnetic ordering. (Although we have chosen to use a ferromagnetic system as a test case, these same selection rules will apply in general, e.g., to paramagnetic and anti-ferromagnetic ordering, and the multiple scattering analysis should be sensitive to differences in the local order of each structure.) To avoid extraneous effects and to allow internal cross-checking of data, measurements were performed only in mirror planes, where only the relative alignment of the photon helicity and magnetization is crucial. Thus reversing the absolute value of these quantities, while maintaining the same relative spin orientation, serves as a convenient but absolutely essential consistency test to determine if the observed asymmetry is due to spin-. dependent diffraction. It is the absence of such polarization control or electron spin-detection, plus the ill-defined nature of the intrinsic $3 \mathrm{~s}$ polarization, that has hampered previous attempts at SPPD using the $3 \mathrm{~s}$ level of $3 \mathrm{~d}$ transition metals ${ }^{12-14}$.

In this paper, we present experimental results for $\mathrm{Fe}$ films on $\mathrm{Cu}(001)$ which demonstrate for the first time spin-dependent photoelectron diffraction using circularly-polarized $x$-rays. Results for scattering along two high symmetry directions demonstrate strong angular effects in the spindependent scattering, and we make the first observation of spin-specific, energy-dependent photoelectron diffraction. Comparison of experimental results with fully spin-polarized multiple scattering calculations demonstrates quantitative agreement. This is the first comparison between experiment and theory for spin-dependent scanned-energy photoelectron diffraction. To facilitate comparison, we introduce a new analytical approach to dichroism asymmetry which removes the peak shift due to the exchange interaction in order to isolate the spin-dependent intensity variations due to diffraction. From this, we have obtained the first detailed and unambiguous structural determination using SFPD.

The experiments were performed at the Stanford Synchrotron Radiation Laboratory using a spherical grating monochromator capable of delivering $\sim 90 \%$ circularly-polarized radiation 15 . Four monolayer (ML) Fe films were grown on a $\mathrm{Cu}(001)$ substrate held at $\sim 150 \mathrm{~K}$. This results in relatively poorly ordered metastable fcc Fe overlayers as evidenced by the diffuse $p(1 \times 1)$ LEED pattern observed for these films. These films have a magnetic easy axis along the sample normal 8,9 , and were magnetized in situ with an electromagnet coil. All measurements were made in remanence. The Fe $2 p$ spectra were collected with an angle-resolving hemispherical 
analyzer with angular acceptance of $\pm 3^{\circ}$. The magnetic axis dictated that the photoelectron polarization is maximized for photons incident along the sample normal. The analyzer position was adjusted to the desired electron emission angle. When the scattering plane contained the sample normal and the (110) azimuth, the electron emission was along the [111] direction. For the (100) azirıucii, the electron emission was along the [110] direction. In all situations, the scattering plane coincided with the orbital plane of the storage ring, i. e., the horizontal plane. Photon energies between 800 and $860 \mathrm{eV}$ were used to give Fe 2p3/2 kinetic energies of 90 to $150 \mathrm{eV}$. Angular alignment was determined using LEED and laser reflection.

Typical Fe 2p spectra for emission along the [110] direction (Figure 1a) and [111] direction (Figure 1b) are shown in Figure 1. These spectra were collected with photon energy hv $=835$ $\mathrm{eV}$ resulting in an electron kinecic energy of $\sim 125 \mathrm{eV}$ at the $\mathrm{Fe} 2 \mathrm{p}_{3 / 2}$ peak. It is important to note that the spectral differences discussed below were dependent only on the relative orientations of photon and minority electron spin and not on any absolute orientation for either quantity. In both sets of spectra the apparent spin-orbit splitting is reduced for the anti-parallel orientation of photon and minority electron spin. This has been observed previously for these films ${ }^{16}$ as well as for $\mathrm{Fe}(001)^{17}$ and is due to the magnetic exchange interaction which shifts the primarily majority spin photoelectrons in the $2 p_{3} / 2$ peak to higher binding energy and the primarily minority spin $2 \mathrm{p}_{1 / 2}$ photoelectrons to lower binding energy. Venus et al. 18 also reported angular variations in the Fe $2 \mathrm{p}$ dichroic asymmetry in angle-resolved photoemission, and attributed this to crystalline symmetry in the photoelectron state. These previous works focused on the dichroic asymmetry arising primarily from the magnetic exchange interaction. We are interested instead in isolating spin-dependent diffraction effects and therefore concentrate on the $\mathrm{Fe} 2 \mathrm{p}$ peak intensity differences which depend only on the spin-polarization of the photoelectrons. Along the [111] direction (Figure 1b), our data demonstrate this spin-dependent intensity difference. It is observed primarily at the $2 p_{3} / 2$ peak for the two electron and photon spin orientations. This is indicative of spin-dependent photoelectron diffraction along this direction. Much smaller intensity asymmetry is observed along the [110] direction (Figure 1a). No intensity asymmetry is observed for an un-magnetized film.

To focus on spin-dependent diffraction effects, it is necessary to separate energy shifts and intensity variations in the spin-orbit split $2 p$ doublet. To remove energy shift effects, we individually align the centroids of both peaks. This is accomplished by shifting the $2 \mathrm{p}_{3} / 2$ peak for the anti-parallel orientation to lower binding energy and the anti-parallel $2 \mathrm{p}_{1 / 2}$ peak to higher binding energy after separating the features in the region between the two peaks. This operation for the [111] direction is displayed in Figure $1 b$ and 1c. Here, Figure $1 b$ is the raw data and 
Figure 1c shows the energy shifts for the anti-parallel data (the spectra are offset for clearer presentation). We note that the spectra are normalized to the low-and high-binding energy backgrounds to extract intensity differences in the $2 p$ photoemission peaks. Because each peak is spin-polarized due to excitation by circularly-polarized $x$-rays, comparing the integrated intensities of peaks collected with parallel and anti-parallel photon and electron spin orientations isolates the spin-dependent diffraction effects.

To determine the intensity asymmetry in the data, we take the difference and divide by the sum of the two spectra on a channel by channel basis. We thereby obtain a spin-dependent intensity asymmetry (SIA) at each binding energy. This results is shown in Figure 1d where the asymmetry varies from $-2 \%$ to $5 \%$. This normalization scheme effectively equates the intensity of the $2 p_{1 / 2}$ features for the two spin orientations and places all the intensity asymmetry in the $2 p_{3} / 2$ peak. The equalization of the $2 \mathrm{p}_{1 / 2}$ features is due in part to the larger background and the Coster-Kronig broadening of this feature ${ }^{19}$. Along the [111] direction a peak asymmetry is consistently observed for the entire photon range examined. The peak asymmetries varied from $\sim 2 \%$ to as high as $\sim 6 \%$. The observed intensity asymmetry is in marked contrast with the fairly isotropic intensity differences that are expected due to mixing of $m_{j}$ states with $2 p_{3 / 2}$ and $2 p_{1 / 2}$ character $^{19}$. The agreement between our experimental measurements and results of spindependent photoelectron diffraction calculations (which will be presented below for both angular and energy dependences of the intensity asymmetry) conclusively demonstrates that the observed intensity asymmetries derive primarily from spin-dependent diffraction.

The theoretical calculation of spin-polarized, multiple-scattering photoemission combines conventional photoemission and spin-polarized low energy electron diffraction (SPLEED) methods ${ }^{20}$. For calculation of the excitation matrix element, $\left\langle\Phi_{E}\left|H^{\prime}\right| \Phi_{E}\right\rangle$, the nonrelativistic approximation is incorporated and the dipole approximation for the interaction Hamiltonian is used. For this study, the Dirac matrix, $\alpha$, is replaced by Pauli matrices, $\sigma$. The selection rules for excitation by circularly-polarized light restrict excitations to $\Delta \mathrm{m}_{j}=+1$ for right-circular polarization and $\Delta \mathrm{m}_{\mathrm{j}}=-1$ for left-circular polarization. The Fe $2 \mathrm{p}$ core-level is split into two sub-levels due to the spin-orbit interaction, and transitions from the sub-levels are governed by these selection rules.

After excitation, the internally-polarized photoelectrons are multiply-scattered inside the crystal in a way similar to spin-polarized LEED electrons. The single site scattering matrix $t^{\mu \mu \mathbf{K}^{\prime}}$ is calculated using the Dirac equation 21 with spin-polarized potentials generated by a selfconsistent linear augmented-plane wave (LAPW) band calculation 22 . This scattering matrix is 
converted to the (lms) representation and used to construct layer diffraction matrices $\mathrm{M}_{\mathrm{gg}}^{\mathrm{ss}}$. After that, the calculation proceeds as in a conventional photoemission calculation 23 except that the dimension of the layer diffraction matrices is doubled to include spin-dependent scattering and spin-flip effects 24 . Note that both spin-orbit coupling and exchange effects are accounted for since off-diagonal matrix elements are nunvanishing. In the calculation, the inner potential is set to $10 \mathrm{eV}$, and inelastic scattering is simulated by an imaginary potential of $4.5 \mathrm{eV}$. Since the electron kinetic energy is relatively low, only terms up to $1=4$ are used for most calculations, but convergence is checked using terms us to $1=6$ with insignificant differences found.

Because the calculated spin-dependent intensities are for an entire $2 p_{3 / 2}$ or $2 p_{1} / 2$ manifold, the best comparison with experiment uses an integrated spin-dependent intensity asymmetry (or integrated SIA) defined as follows:

Integrated SIA (hv) $=\frac{\left[\frac{I_{3 / 2}^{+}(h v)}{I_{1 / 2}^{+}(h v)}-\frac{I_{3 / 2}^{-}(h v)}{I_{1 / 2}^{-}(h v)}\right]}{\left[\frac{I_{3 / 2}^{+}(h v)}{I_{1 / 2}^{+}(h v)}+\frac{I_{3 / 2}^{-}(h v)}{I_{1 / 2}^{-}(h v)}\right]}$

where, for example, $I_{3 / 2}^{+}$is the parallel intensity integrated over the $2 \mathrm{p}_{3 / 2}$ manifold. In Figure 2, we show the calculated asymmetry (solid curve) along the [111] direction for a model with fccFe spacings $\left(d_{12}=1.8 \AA, d_{23}=1.8 \AA\right)^{14}$ as a function of photon energy compared to the asymmetry extracted from the experimental data. In order to obtain an integrated SIA from our experimental data, the SIA curves (like that shown in Figure 1d) are integrated over a $10 \mathrm{eV}$ window centered at the 2 p3/2 peak. The integrated SIA gives a good quantitative agreement with the calculations and even reproduces the sign change in the asymmetry ca. hv $=840 \mathrm{eV}$. Extending the integration limits will lower the asymmetry curve, but the choice of limits is not completely unrestricted due to the mixing of states with $2 \mathrm{p}_{3} / 2$ and $2 \mathrm{p}_{1 / 2}$ character. However; the qualitative trends are all reproduced independent of the integration range and background parameters. The energy oscillations due to spin-dependent photoelectron diffraction are fundamental and are present even in the peak height intensities. Furthermore, variations of the structural parameters by $\pm 0.2 \AA$ destroys the agreement between the measurements and simulations. In fact, we have performed a detailed structural determination by varying the spatial parameters in the model and performing a quantitative $r$ factor analysis of calculated and measured values. Our best fit occurs for $d_{12}=1.9 \AA$ and $d_{23}=1.7 \AA$ (dashed curve), with a Pendry $r$ factor ( $r_{p}$ ) of 0.17 , which is $9 \%$ better than the unrelaxed structure, a measurable difference. This is consistent with our previous 
determination ${ }^{14}$ but exceeds those earlier results with a clear improvement in sensitivity to the small surface relaxations. Thus, the agreement between theory and experiment indicates that the intensity asymmetry arises from spin-dependent diffraction.

Nev:, we return to the angular dependences in the SIA. The calculations have been done for scattering of $125 \mathrm{eV}$ kinetic energy electrons (i.e., $\mathrm{hv}=835 \mathrm{eV}$ ). This ISIA value is $0.27 \%$ for emission along the [110] direction. This value is about four times lower than that for emission along the [111] direction and is again consistent with the qualitative trends in the experimental data. It is important to note that identical calculations omitting final state diffraction events do not reproduce the energy and angular variations in the SIA. Additionally, because our measurements are made in mirror planes, other dichroic effects vanish 25 . (These effects also depend on the absolute photon helicity and not the relative spin orientations. Thus the internal cross-check of varying both quantization axes is critical in isolating the SPPD effect.) Thus the agreement in both the angular and energy variations of the experimental and calculated intensity asymmetries demonstrates that the underlying cause of this intensity asymmetry is spindependent photoelectron diffraction.

In summary, we have for the first time measured spin-dependent photoelectron diffraction using circularly-polarized $x$-rays. The resultant spin-polarized $2 p$ photoemission peaks exhibit angle and energy dependent intensity variations due to spin-dependent final state diffraction. The results are well described by spin-polarized photoemission, multiple scattering calculations:

Work performed under the auspices of the U.S. Department of Energy by the Lawrence Livermore National Laboratory under contract number W-7405-ENG-48. SSRL is supported by the Chemical Sciences Division of DOE/BES. The work at the University of WisconsinMilwaukee was supported by DOE DE-FG02-84ER45076. 


\section{Figure CAPTIONS}

Figure 1: $\mathrm{Fe} 2 \mathrm{p}$ spectra for $4 \mathrm{ML} \mathrm{Fe} / \mathrm{Cu}(001)$ along the [110] direction (a) and [111] direction (b) for parallel (solid curves) and anti-parallel (dashed curves) photon and minority electron spin orientation. The energy shifts are due to the magnetic exchange interaction of the spin-polarized photoelectuns Note the intensity variations in the $2 p_{3} / 2$ peak for the [111] direction and its absence along [110]. (c) A demonstration of how the peaks are shifted to remove exchange effects from the comparison of intensities. (d) Intensity asymmetry derived from the spectra in (b). The 2p3/2 peak asymmetry is $\sim 5 \%$. The data in the pre-edge region (binding energy $<705 \mathrm{eV}$ ) was smoothed to reduce noise in the difference spectra in this region.

Figure 2: Calculated (solid curve: $d_{12}=d_{23}=1.8 \AA, r_{p}=.19$; dashed curve: $d_{12}=1.9 \AA, d_{23}=$ $1.7 \AA, r_{p}=0.17$ ) and measured intensity asymmetries along the [111] direction as a function of photon energy. The oscillatory behavior in the curves is due to spindependent photoelectron diffraction. Representative error bars are included with the experimental data, shown as discrete values (triangles). See text for details. 


\section{REFERENCES}

1. S.S.P. Parkin, Phys. Rev. Lett. 71, 1641 (1993).

2. B.P. Gurney, V.S. Speriosu, J.P. Nozieres, H.F. Lefakis, D.R. Wilhoit, and D.U. Need, Phys. Rev. Lett. 71, 4023 (1993).

3. J.G. Tobin, G.D. W.aldill. Hua Li, and S.Y. Tong, Phys. Rev. Lett. 70, 4150 (1993).

4. J.G. Tobin, G.D. Waddill, and D.P. Pappas, Phys. Rev. Lett. 68, 3642 (1992).

5. S.A. Chambers, T.J. Wagener, and J.H. Weaver, Phys. Rev. B 36, 8992 (1987).

6. D.A. Steiger wald and W.F. Egelhoff, Phys. Rev. Lett. 60, 2558 (1988).

7. D. Pescia, M. Stampanoni, G.L. Bona, A. Vaterlaus, F. Meier, G. Jennings, and R.F. Willis, Phys. Rev. Lett. 60, 2559 (1988).

8. D.P. Pappas, K. -P. Kamper, and H. Hopster, Phys. Rev. Lett. 64, 3179 (1990).

9. C. Liu, E.R. Moog, and S.D. Bader, Phys. Rev. Lett. 60, 2422 (1988).

10. D.D. Chambliss, R.J. Wilson, and S. Chiang, J. Vac. Sci. Technol. A10, 1993 (1993); D.D. Chambliss, et. al., Mat. Res. Soc. Symp. Proc. 313, 713 (1993).

11. G. Schütz, et. al., Phys. Rev. Lett. 62, 2620 (1989).

12. F.Z. Hang, et al., Bulletin APS $\underline{39}, 904$ (1994), R27.4; B. Sinkovic and C.S. Fadley, Phys. Rev. B31, 4665 (1985); B. Sinkovic, et al., Phys. Rev. Lett. 55, 1227 (1985); B. Hermsmeier, et. al., Phys. Rev. Lett. 62, 478 (1989).

13. M.T. Johnson, H.I. Starnberg, and H.P. Hughes, J. Phys. Cㅁㅇ, 4385 (1987).

14. J.G. Tobin, M.K. Wagner, X.Q. Guo, and S.Y. Tong, Mat. Res. Soc. Symp. Proc. 208, 283 (1991).

15. K.G. Tersell and V.P. Karpenko, Nuc. Instrum. Meth. Phys. Res., Section A 291, 511 (1990); L.J. Terminello, G.D. Waddill, and J.G. Tobin, ibid. 319, 271 (1992).

16. G.D. Waddill, J.G. Tobin, and D.P. Pappas, Phys. Rev. B 46 , 552 (1992).

17. L. Baumgarten, et. al., Phys. Rev. Lett. 65, 492 (1990).

18. D. Venus, et. al., J. Phys. 5, 1239 (1993).

19. H. Ebert, et. al., Phys. Rev. B 44, 4406 (1991).

20. R. Feder, in Polarized Electrons in Surface Physics, R. Feder, ed., (World Scientific, Singapore, [1985]).

21. R. Feder, F. Rosicky, and B. Ackerman, Z. Phys. B $\underline{52}, 31$ (1983).

22. H.J. Jansen and A.J. Freeman, Phys. Rev. B $\underline{30}, 561$ (1984).

23. C.H. Li, A.R. Lubinsky, and S.Y. Tong, Phys. Rev B 17, 3128 (1978).

24. A. Orneci, B.M. Hall, and D.L. Mills, Phys. Rev. B $\underline{41}, 4524$ (1990).

25. H.P. Oepen, K. Hunlich, and J. Kirschner, Phys. Rev. Lett. $\underline{56}, 496$ (1986). 


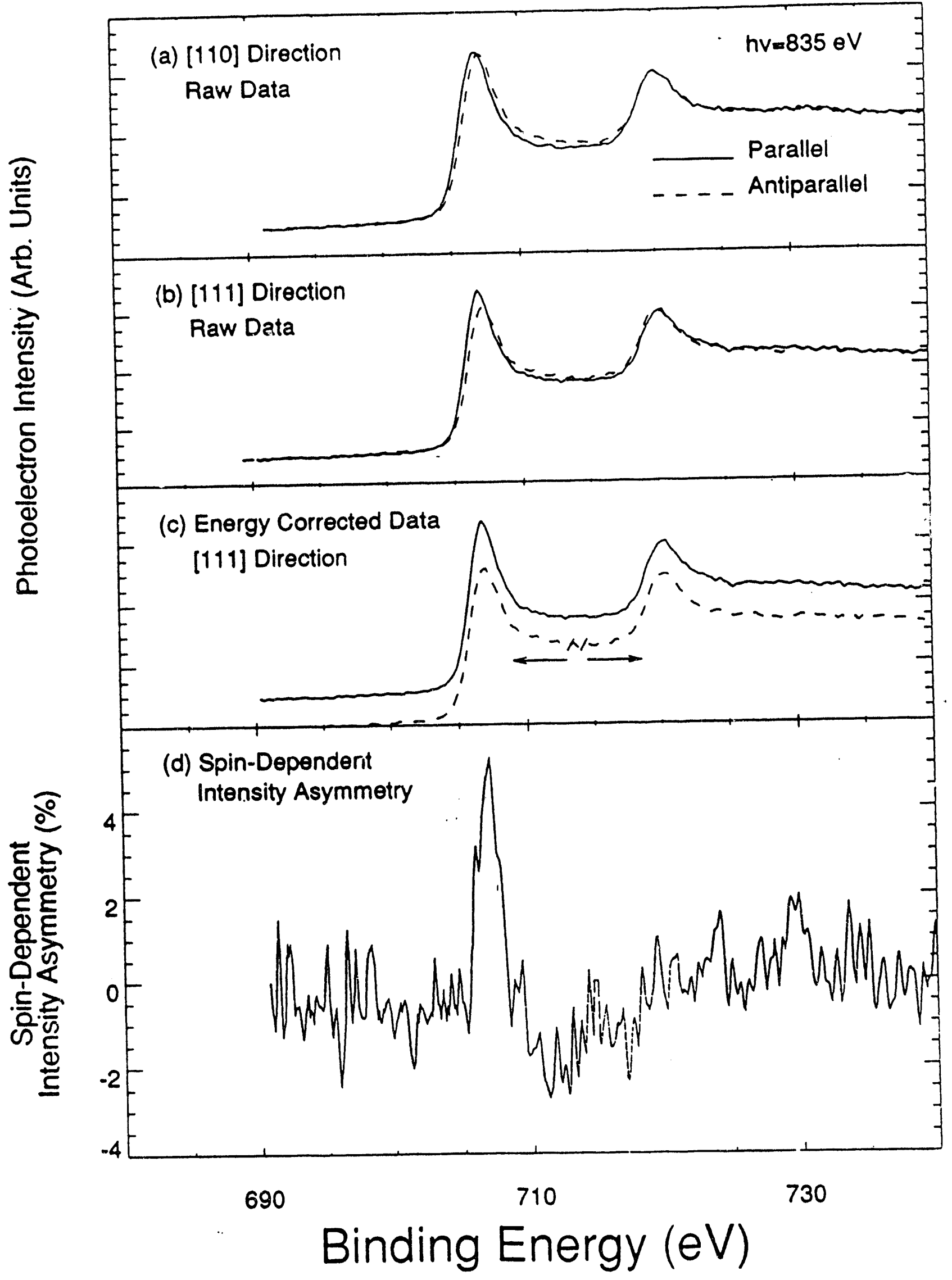

Figure 1 


\section{Integrated SIA (\%)}

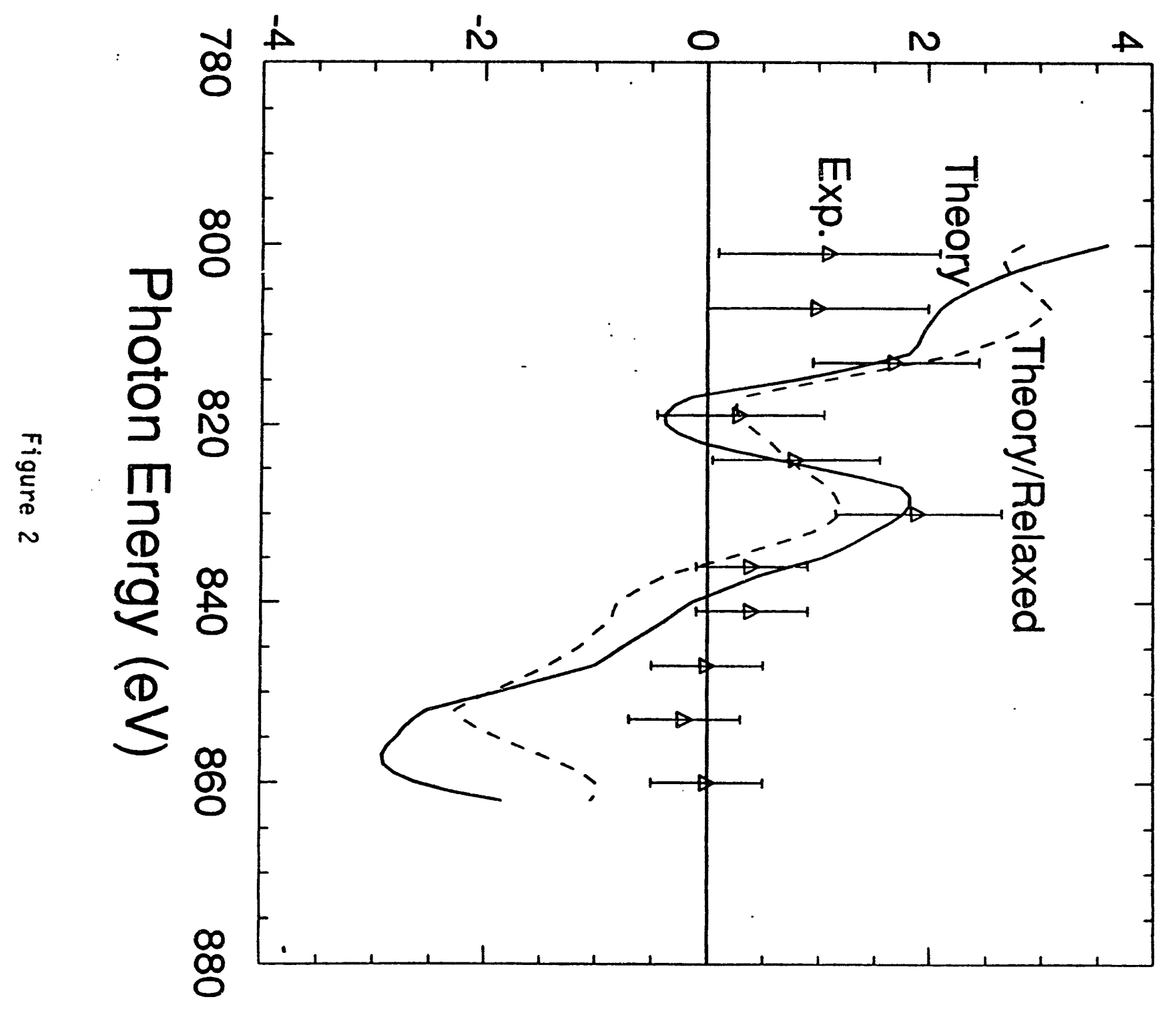



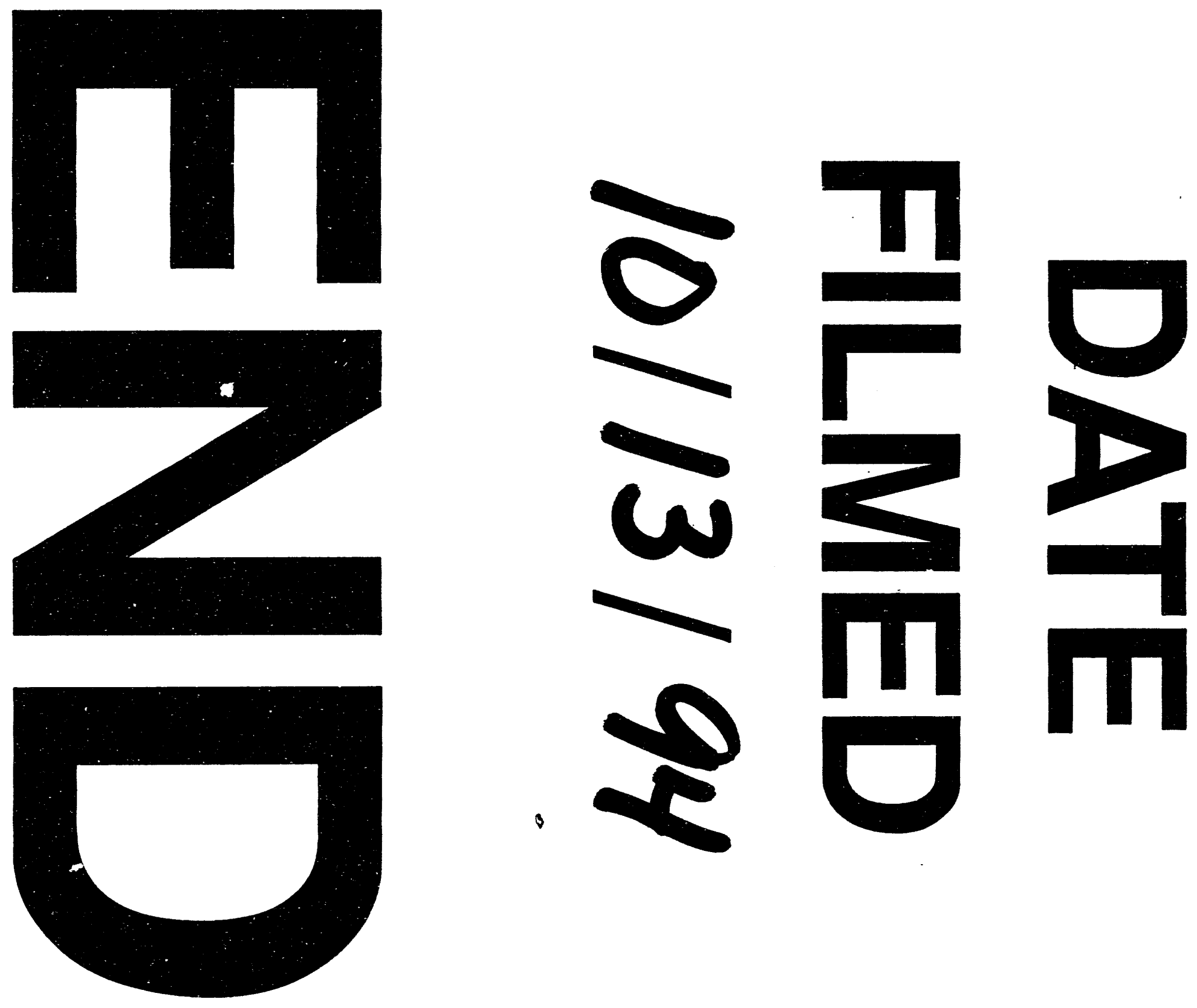

$\checkmark$ 
